\title{
L'EDUCAZIONE DELLE RAGAZZE NEL XIV SECOLO SECONDO FRANCESCO DA BARBERINO ${ }^{1}$
}

Lo scopo di quest'articolo è di presentare le virtù che dovevano avere le bambine e poi le ragazze in età di matrimonio, provenienti da famiglie di alto status sociale, secondo l'opera di Francesco di Neri da Barberino intitolata Reggimento e costumi di donne. Quest'opuscolo scritto nel XIV secolo da un notaio e poeta italiano è uno dei pochi trattati medievali indirizzato esclusivamente al pubblico femminile. Infatti gli autori di quest'epoca rivolgevano le loro opere soprattutto agli uomini perché da una parte erano ritenuti più intelligenti delle donne e d'altra parte avevano più possibilità di ricevere una buona istruzione. Invero gli uomini del tempo si concentravano di più sull'educazione dei figli lasciando il compito della formazione delle ragazze ai monasteri, o alle loro madri.

Il futuro della figlia dipendeva dai suoi genitori, soprattutto dal padre. Infatti erano loro a decidere se la bambina dovesse abbracciare la vita religiosa o diventare moglie. Presa la decisione, la ragazza, la cui sorte era legata alla chiesa, veniva mandata in monastero dove imparava tutto il necessario per farsi monaca. Invece, come si vedrà in seguito, se la bambina in futuro doveva maritarsi era inviata o alla scuola di un convento o alla corte di una nobile donna o infine rimaneva a casa dove imperava tutto ciò che le era necessario per diventare una buona moglie ${ }^{2}$.

Trattando dell'educazione femminile nel medioevo non si deve pensare che assomigliasse molto a quella maschile. In realtà alle ragazze non si insegnavano le materie di trivium e quadrivium. I mariti non aspettavano dalle loro mogli questa conoscenza. Infatti gli uomini non si preoccupavano troppo dell'istruzione delle donne, considerandola spesso una perdita di tempo e di denaro. D'altronde nell'istruzione femminile vedevano un grande pericolo. Infatti temevano un malinteso o inganno, poiché se la donna fosse stata capace di leggere e di scrivere allora avrebbe potuto leggere romanzi o testi di teologia, di filosofia o altri, che avrebbero potuto essere da loro male interpretati. Inoltre all'insaputa del padre o del marito la donna avrebbe potuto, scrivere lettere d'amore al suo amato ${ }^{3}$. Non stupisce allora che la maggior parte degli uomini ritenesse più sicuro far imparare alle loro figlie

\footnotetext{
1 Cfr. E. Pasquini, Francesco da Barberino in Dizionario Biografico degli Italiani, Catanzaro 1997, pp. 686-691; R. Ortiz, Francesco da Barberino e la lettura didattica neolatina, Roma 1948, pp. 19-24.

2 Cfr. E. Power, Donne nel medioevo, a cura di M. M. Postan, Milano 19994, p. 75, P. Aries, Padri e figli nell'Europa medioevale e moderna, vol. 2, Bari 1976, p. 430.

3 Cfr. E. Power, L'educazione delle donne, in: Né Eva né Maria. Condizione femminile e immagine della donna nel Medioevo, a cura di M. Pereira, Zanichelli, Bologna 1981, p. 103.
} 
cose come ad esempio guidare la casa, badare ai fratelli più piccoli, cucire o filare evitando l'apprendimento della lettura e scrittura.

Prima di presentare il pensiero di Francesco da Barberino riguardo all'istruzione della ragazza quando comincia bene e mal sentire, e vergogna temere 4 e poi quando in tempo verrà di maritaggio ${ }^{5}$ si ritiene necessario trattare brevemente dell'educazione delle ragazze. Inoltre si vogliono riportare i titoli di alcune opere di contenuto educativo del tempo, dedicate sia alle donne che agli uomini, visto che l'opuscolo di Barberino non era l'unico testo di questo genere scritto durante l'epoca di mezzo.

\section{L'istruzione delle donne e i trattati educativi}

È un compito abbastanza complesso trattare oggi dell'educazione femminile nel medioevo. A motivo della scarsità di testi d'epoca è difficile dire con certezza come si presentava in quel periodo l'istruzione della donna. Infatti una studiosa italiana, Angela Giallongo, in un suo libro dedicato all'educazione nel medioevo ha sottolineato che la bambina è la grande assente nella storia dell'educazione ${ }^{6}$. Grazie però alle fonti pervenute ai nostri tempi si possono notare due linee d'istruzione femminile. La prima riguarda l'educazione cortese mentre la seconda l'istruzione della donna sul modo di occuparsi del marito7.

Al centro dell'educazione cortese c'era l'insegnamento delle buone maniere che dovevano essere ben conosciute da tutte le donne che volevano o dovevano brillare in società. Tuttavia esse oltre al galateo imparavano tra l'altro a leggere, raccontare novelle, andare a caccia, giocare a scacchi o gli altri giochi di società e così via ${ }^{8}$. Robert de Blois, un poeta francese del XIII secolo, in una delle sue poesie lodava le abilità di una signora:

Portava e faceva volare il falcone, il terzuolo e il falco, sapeva giocar bene a scacchi e a tavola reale,

leggere novelle e raccontarle, cantare canzoni. Tutto ciò che una signora ben allevata doveva sapere, lei lo sapeva, e non gliene mancava nessuna ${ }^{9}$

Il secondo modello educativo riguardava invece il comportamento della moglie verso il marito, i suoi obblighi nel governare la casa, i doveri religiosi e altro ancora ${ }^{\mathrm{I}}$. Ci sono pervenuti alcuni testi, come ad esempio quello di Menager de Paris di XIV secolo, intitolato Come una buona moglie insegna alla figlia ${ }^{\mathrm{II}}$, o quel testo inglese anonimo, probabilmente anch'esso del XIV secolo, intitolato Come la buona madre insegnò alla figlia ${ }^{12}$ che dimostrano quali aspettative avevano i mariti e quali virtù dovevano avere le mogli.

Francesco da Barberino, Reggimento e costumi di donne, a cura di G. E. Sansone, Roma 1995, p. 6.

Ibidem.

A. Giallongo, Il bambino medievale. Educazione ed infanzia nel Medioevo, Bari 1997, p. 26.

Cfr. E. Power, L'educazione delle donne, op. cit., p. 102-103.

Cfr. Idem, Donne nel medioevo, op. cit., p. 72.

Ibidem.

10 Cfr. Ibidem, pp. 72-73.

11 Menager de Paris, The good wife's guide, G. L. Greco, Ch. M. Rose translated with Critical Introduction, Cornell University 2009.

12 Anna di Francia, Come la buona madre insegnò alla figlia in C. Xodo Cegolon, Lo specchio di Margherita, per una storia dell'educazione femminile nel Basso Medioevo, C.L.E.U.P., Padova 1988, p. 130-137. 
Dai testi scritti nell'epoca medievale pervenuti ai nostri giorni si osserva che non esisteva un concetto omogeneo riguardo all'insegnamento delle donne a leggere e scrivere. Invero, alcuni scrittori, come Francesco da Barbarino, ritenevano che soltanto le ragazze provenienti da case nobili dovessero sapere leggere e scrivere. Queste capacità, secondo il poeta, potevano essere utili in futuro per occuparsi delle loro proprietà. D'altra parte, lo stesso Barberino dubitava se questa istruzione fosse necessaria per le figlie del semplice signore, ma sicuramente doveva essere proibita alle figlie dei mercanti e degli artigiani ${ }^{\mathrm{I} 3}$. Riguardo al leggere Anna di Francia nel suo Insegnamento alla figlia Susanna, consigliandole la lettura di alcuni autori scrisse: Perché voi sappiate meglio vivere e condurvi in devozione vi consiglio di leggere il libretto del saggio S. Luigi, quello di S. Pietro di Lussemburgo, le somme e la regola, l'orologio di Sapienza o altri libri della vita dei santi, anche i detti dei filosofi e degli antichi saggi, le cui dottrine devono essere per voi retta regola ed esempio, e questa è onestissima occupazione e passatempo ${ }^{\mathrm{I4}}$. Un altro sguardo circa il sapere leggere e scrivere ci danno Menagier di Parigi e Cristina di Pisan che raccomandavano l'insegnamento a tutte le donne ${ }^{15}$. La stessa opinione aveva anche il Cavaliere de la Tour Landry, il quale raccomandava l'apprendimento della lettura, dicendo che la donna aveva il dovere di saper leggere le Scritture ${ }^{16}$. D'altra parte Paolo da Certaldo diceva ad un padre che non ista troppo bene ad una femina sapere leggere se già no la volessi fare monaca ${ }^{17}$. C'erano poi gli scrittori, come Filippo di Navarra, i quali sostenevano che l'educazione dovesse essere proibita a tutto il sesso femminile ${ }^{18}$.

Riguardo all'educazione delle ragazze di famiglie nobili c'erano tre modi d'apprendere un'istruzione adeguata per il loro casato. Infatti esse potevano studiare nelle scuole di un convento, nella corte o nelle case di qualche grande signora o infine rimanere e imparare il necessario a casa propria ${ }^{19}$. Tutto dipendeva dalla situazione economica della famiglia. Riguardo all'insegnamento nelle scuole dei conventi non si può dire molto. Sfortunatamente non ci sono pervenuti documenti che potrebbero gettare luce sulle materie insegnate. Sicuramente le ragazze imparavano a leggere, a scrivere il catechismo e il latino, almeno fino al XIV secolo ${ }^{20}$, ma non si sa altro. Come sottolinea Eleen Power nei primi secoli del medioevo il livello intellettuale in molti conventi era elevato, ma con il tempo peggiorò. Infatti già nel XIV secolo, come è stato già detto, non si insegnava più latino, e dal XV secolo si abbandonò l'insegnamento del francese ${ }^{21}$. Inoltre l'educazione nelle scuole dei conventi era costosa anche per le famiglie nobili e non tutti i genitori potevano permettersi di mandarvi le loro figlie, visto che spesso non si trattava di una ma di due, tre o più ragazze.

Un secondo modo di apprendere le materie adatte alle figlie di case nobili era mandarle a corte reale o a casa di qualche nobile donna. Infatti anche Anna di Francia negli Insegnamenti

13 Cfr. E. Power, Donne nel medioevo, op. cit., p. 74; Idem, L'educazione delle donne, op. cit., p. 103.

14 Anna di Francia, Insegnamenti alla figlia Susanna, op. cit., p. 89.

15 Cfr. E. Power, Donne nel medioevo, op. cit., p. 74.

16 G. Tour De La Landry, The Book of the Knight of La Tour-Landry: Compiled for the Instruction of His Daughters, London 1868, p. 117.

17 Paolo da Certaldo, Libro di buoni costumi, a cura di A. Schiaffini, Firenze 1945, p. 27.

18 Cfr. E. Power, Donne nel medioevo, op. cit., p. 74.

19 Cfr. Idem, p. 75; P. Aries, Padri e figli, op. cit., p. 430.

20 Cfr.. E. Power, Donne nel medioevo, op. cit., p. 77.

21 Cfr. Ibidem, pp. 76-77. 
alla figlia Susanna consiglia alla ragazza di mettersi al servizio di dama o damigella che goda di buona reputazione, non sia volubile ed abbia buon senso ${ }^{22}$. Invero qui le bambine passavano molte ore in compagnia della signora, e osservandola e servendola imparavano tutto ciò che poteva essere loro utile in futuro. Inoltre alla corte c'erano più possibilità di incontrare uomini influenti e di sposarsi con un buon partito. Infatti le famiglie speravano che oltre allo studio del galateo e di altre cose le ragazze facessero un buon matrimonio.

Il terzo modo, e forse anche quello più comune, era lasciare le ragazze a casa e far loro imparare le cose dalla madre e, se ci fossero stati fratelli, dall'insegnante, assunto dal padre per loro. Infatti accadeva che le ragazze, se il padre era d'accordo, oltre all'insegnamento della madre ricevevano istruzione dal tutore incaricato di educare i ragazzi. In questo modo le fanciulle imparavano dalla madre il necessario che dovevano sapere e poi per qualche ora imparavano con i fratelli le materie insegnate dal tutore.

Nella maggioranza dei casi non si aspettava dalle ragazze nobili, come è stato già detto, la conoscenza delle materie di trivium o quadrivium, ma piuttosto di conoscere il galateo, di raccontare le storie, di conoscere i giochi di società, etc. Ovviamente non si può generalizzare perché c'erano donne che avevano ricevuto una buona educazione, come ad esempio Duoda, l'autrice di un libretto scritto per il figlio Guglielmo, Trotula la medichessa di Salerno, Eloisa l'amata di Abelardo o Christine de Pisan l'autrice di tante opere. Si nota subito che sono stati elencati soltanto i nomi delle donne laiche, e tralasciate quelle delle monache. Infatti le ragazze, entrando in un monastero, ricevevano l'educazione adeguata per una monaca che non dipendeva più dai loro genitori.

Riguardo all'educazione delle ragazze che in futuro dovevano sposarsi, si deve dire che esse imparavano le materie adeguate al loro status sociale. Infatti era ovvio che le principesse imparassero cose differenti da quelle che si richiedevano ad esempio dalle figlie di un mercante. D'altra parte non si notava molta differenza nelle materie insegnate e nell'esigenza del comportamento delle ragazze di famiglie nobili. Francesco da Barberino infatti sottolineava che dalle figlie di un barone o di un conte si aspettavano costumi simili a quelli delle figlie dell'imperatore $\mathrm{o}$ del $\mathrm{re}^{23}$. Inoltre la società era più esigente riguardo all'atteggiamento delle ragazze di famiglie nobili che di quelle di un mercante o un contadino. Si lasciava più libertà alle ragazze di classi sociali più basse, mentre a quelle nobili si guardava con più severità. Questa diversità si nota subito nell'opera di Barberino, che nel Reggimento $e$ costumi di donne si rivolge alle ragazze distinguendole non soltanto secondo la loro età ma anche secondo il loro casato. Infatti l'autore tratta delle fanciulle, donzelle, donne maritate, vedove, religiose, cameriere, serve, balie, schiave e donne nei vari mestieri. Inoltre le distingue seguendo i livelli sociali cominciando dalle figlie dell'imperatore, di un re incoronato, barone, cavaliere, mercante, etc.

In questo luogo si ritiene opportuno sottolineare che l'opera di Francesco da Barberino non era l'unico testo con indicazioni educative scritto in quest'epoca. Una cosa però che distingue il Reggimento da molte opere di quel periodo è questa che l'opuscolo è esclusivamente dedicato al pubblico femminile. Infatti la maggior parte dei testi era indirizzata agli uomini come testimonia nel proemio l'autore del Reggimento: Novellamente, Francesco,

22 Anna di Francia, Insegnamenti alla figlia Susanna, op. cit., p. 91.

23 Cfr. Francesco da Barberino, Il Reggimento, op. cit., p. 23. 
parlai coll'Onestade e, a preghiera di molte altre donne, mi lamentai con lei, e dissi ch'erano molti ch'aveano scritti libri, costumi ornati d'omo, ma non di donna ${ }^{24}$.

Un altro testo che tratta di bon ton è il Libro di buoni costumi di Paolo da Certaldo e il Livre des trois vertus di Christine de Pisan. Inoltre Bonvesin da Riva scrisse De quinquaginta curialitatibus ad mensam. C'era anche un altro testo, sfortunatamente perduto, intitolato Della Cortesia di Tommasino dei Cerchiari. Del comportamento ed educazione delle giovani donne parlava anche nelle sue prediche uno dei più famosi predicatori italiani del XV secolo: Bernardino di Siena ${ }^{25}$. Oltre ai testi di cortesia ci sono pervenuti anche testi che davano istruzioni riguardo al matrimonio come ad esempio l'opera di Menagier de Paris Come una buona moglie insegna alla figlia o il testo anonimo inglese Come una madre insegnò alla figlia.

\section{Il Reggimento e l'educazione}

\subsection{La Fanciulla}

Il Reggimento e costumi di donne di Francesco da Barberino scritto sia in versi che in prosa è composto di un proemio e di venti capitoli. Il primo capitolo dedicato alle ragazze in età nella quale esse cominciano a vergognarsi e arrossire ${ }^{26}$ l'autore, all'inizio, si rivolge alle fanciulle provenienti dalle famiglie di alto status sociale, come le figlie di un imperatore e di un re per passare poi alle figlie di un conte, barone, cavaliere, medico, mercante etc.

Riguardo alle figlie di imperatore, di un re, di un conte o di un barone e cavaliere, la società aveva aspettative molto alte. Infatti si pretendeva da loro un comportamento impeccabile. Invero esse dovevano essere ben educate, conoscere il galateo, sapersi trovare in qualsiasi circostanza e situazione. Viste le grandi aspettative le ragazze già da piccole imparavano dalle loro madri, balie e insegnanti le buone maniere e un comportamento corretto. Per evitare qualsiasi errore nell'atteggiamento delle ragazze si riteneva necessaria una continua presenza della madre o delle altre donne di casa. Infatti la loro compagnia, ma soprattutto quella della madre, la esigeva anche Barberino ${ }^{27}$.

Riguardo alla vita sociale delle ragazze in questa età si preferiva tenerle lontane dalla gente e soprattutto dagli uomini. Il più grande predicatore Senese, Bernardino, diceva che le ragazze dovrebbero essere chiuse dentro la casa perché non ci è meglio che stare rinchiusa ${ }^{28}$ per dedicarsi, se sapevano leggere, alla lettura de le cose Sante e buone"29 o a qualsiasi lavoro utile. Se la ragazza era presente tra gli ospiti dei genitori dove incontrava i cavalieri, questo accadeva soltanto se era invitata dal padre, dalla madre o da qualche persona di casa, ma doveva essere sempre accompagnata dalla sua balia o maestra ${ }^{30}$. La ragazza non poteva rimanere con gli uomini da sola senza la compagnia di altre donne. Inoltre doveva essere

\footnotetext{
24 Ibidem, p. 1.

25 Bernardino da Siena, Le prediche volgari: quaresimale fiorentino 1424, a cura di C. Cannarozzi vol. 2, A. Pacinotti, Pistoia 1934; idem, Le prediche volgari, vol. 3, Firenze 1940; idem, Le prediche volgari, vol. 2, Firenze 1958; idem, Prediche volgari sul campo di Siena 1427, a cura di C. Delcorno, vol. 2, Milano 1989.

26 Francesco da Barberino, Reggimento, op. cit., p. 9.

27 Cfr. Ibidem, p. 9.

28 Bernardino da Siena, Prediche volgari sul Campo di Siena 1427, a cura di C. Delcorno, vol. 2, Rusconi, Milano 1989 , p. 862.

29 Ibidem, p. 862.

30 Francesco da Barberino, Reggimento, op. cit., p. 10.
} 
attenta a non guardarli, perché fissando un uomo questo poteva facilmente illudersi di essere amato da lei e di sentirsi obbligato a corteggiarla ${ }^{31}$.

Oltre a questo quando la ragazza si trovava tra la gente doveva ricordarsi di sapere quando parlare e quando tacere:... non è sì da tacere che altri non parli mai, sì ch'altri non dicesse: Ella non parla perch'ella è muta ${ }^{32}$. Infatti la fanciulla doveva trovare un equilibrio, ma era ben vista quando ascoltava più che quando parlava. Un'altra cosa da evitare era la gesticolazione durante il colloquio. Il poeta sottolineava infatti s'ella è domandata o mandata a parlare, rispondi e parli temperamente e 'l suo parlare sia basso, colle sue mani e l'altre membra ferme ${ }^{33}$. La stessa opinione condivideva Bernardino da Siena che in una delle sue prediche rivolgendosi ai genitori diceva che dovevano insegnare alla figlia a non essere mai una "parlatrice" 34 e di ricordarsi il proverbio chi troppo parla, spesso falla" . Anche Anna di Francia raccomandava alla figlia di parlare e guardare con moderazione e di non essere prima né l'ultima in una conversazione ${ }^{36}$.

Riguardo al comportamento alla mensa Barberino insegnava che la ragazza doveva essere sempre ordinata e cortese ${ }^{37}$. L'autore del Reggimento sottolineava che non era ben visto quando una ragazza beveva poiché quanto che nell'uomo l'ebriare stia male, sta nella donna troppo più villano ${ }^{38}$. Un'altra regola riguardava la conversazione durante i pasti. Infatti essa non doveva parlare eccessivamente né appoggiare la testa sulle mani e semmai parla poco, questo è quello luogo dove le conviene allora men parlare né mai si tenga il capo colle mani, né giaccia s'ella è sana in collo a sua maestra ${ }^{39}$.

Barberino non vedeva niente di male nell'insegnamento del canto e del ballo. Infatti permetteva alla ragazza di cantare quando si trovava tra la gente, ma soltanto sulla esplicita richiesta del signore, della madre o delle sue compagne. Lei non doveva però esaudire subito la loro richiesta, perché era ben vista se resistesse un po' alle loro preghiere ${ }^{40}$. Inoltre durante il canto doveva ricordarsi di non guardare la gente ma di avere gli occhi chinati e stando volta a chi magior vi siede ${ }^{4 \mathrm{I}} \mathrm{Il}$ poeta assicura che questo canto chiamato camerale piace molto e passa ne' cuori ${ }^{42}$.

Barberino concedeva alla ragazza il ballo ma lo doveva fare onestamente ${ }^{43} \mathrm{e} \mathrm{senza}$ saltare troppo per evitare la situazione di Sensonia, figlia di messer Guglielmo da Folcalchieri che durante il ballo mostrò la gamba ${ }^{44}$.

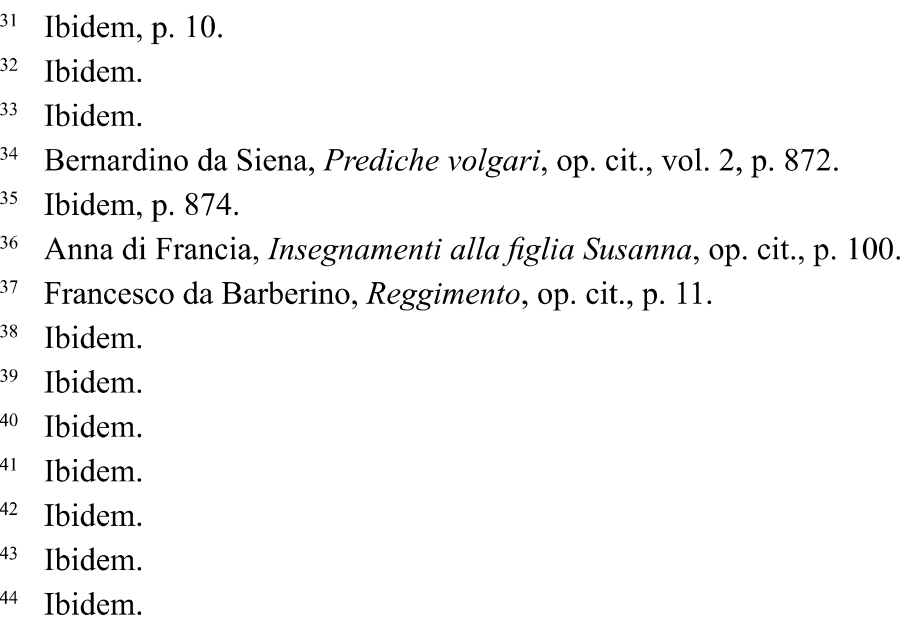


Barberino esigeva dalla ragazza la modestia anche nel pianto e nel riso. Infatti essa poteva piangere, ma il suo pianto doveva essere sanza voce ${ }^{45}$. Riguardo al riso non era di buon gusto ridere in modo tale di far vedere i denti: non gridi a! a! né con simili voci, né però che ciò faria mostrar li denti che non è cosa conta; ma senza alcun rumore ${ }^{46}$. Lo stesso condivideva Anna di Francia la quale insegnava a Susanna non ridete troppo, qualunque sia la causa, perché è molto sconveniente, massimamente per le giovani nobili, le quali, in ogni circostanza, devono avere modi più sobri, dolci e sicuri delle altre ${ }^{47}$.

Circa l'ornamento della ragazza Barberino non vedeva niente di male e diceva lodo che si sforzi e piaccia a lei lo bene andare acconcia e se ghirlanda porta, lodo che sia pure una gioliva e picoleta ${ }^{4}$. Però per la fanciulla era necessario trovare un rimedio e non ornarsi troppo. Infatti doveva tenere a mente che l'addobbo migliore è la natura stessa:

Piacemi in donna bellezza che dura

e quella è da natura ${ }^{49}$.

Un po' più luce riguardo al modo di vestirsi e di ornarsi delle ragazze danno Bernardino da Siena e Anna di Francia. Infatti il predicatore Senese riteneva un peccato avere troppi capi d'abbigliamento ${ }^{50}$. Inoltre insisteva che esso mostrasse l'onestà della giovane donna ${ }^{51}$. Al riguardo parlava di più Anna di Francia quando insisteva sul vestirsi bene e in modo pulito. Infatti raccomandava a sua figlia Susanna fate sempre in modo da vestirvi meglio che potete, nel modo più pulito $^{52}$. Per lei era sconveniente e assai disonesto vedere una ragazza o donna nobile graziosamente abbigliata e non curata ${ }^{53}$. Inoltre Anna insisteva di non portare vestiti che rechino oltraggio, né troppo stretti né troppo cadenti ${ }^{54}$.

Per di più, secondo Barberino, la ragazza andando in chiesa per la messa doveva essere sempre in compagnia e comportarsi modestamente. E se colla sua madre forse alla chiesa andasse, a poco a poco imprenda di stare onesta e conta, e ad orare e paternostri dire, come la madre vede e l'altre donne stare, sempre seguendo l'amaestramento della sua balia o balio, in quanto egli è laudevole e onesto ${ }^{55}$.

L'autore di Reggimento riguardo all'insegnamento di leggere e di scrivere diceva: E parmi ch'a suo stato si convenga che in questo tempo imprenda legere e scrivere convenevolmente, sicché, se convenisse lei donna rimanere di terra o di vassalli, sarà più conta a reggimento fare; ché ben saver che il senno accidentale, lo qual porrà poi conquistar legendo, aiuta il naturale in molte cose $e^{56}$. Il poeta riteneva il saper leggere una cosa utile che avrebbe potrebbe

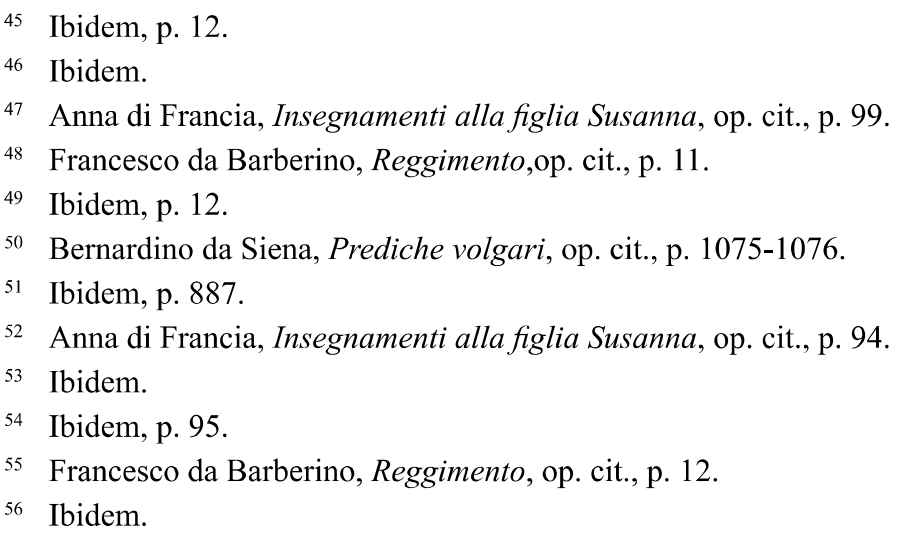


servirle in futuro, ma suggeriva che l'insegnante di queste materie doveva essere o una donna o tale persona che non sia suspetta ${ }^{57}$.

\subsection{La donzella}

Il secondo capitolo del Reggimento Barberino lo dedica alle ragazze in età di matrimonio. Esse dovevano ricordarsi tutto ciò che avevano imparato finora e di essere attente a non rovinare la loro reputazione.

Una delle cose più importanti per il poeta era quella di tenere le ragazze che erano in quest'età lontane dalle finestre, balconi, chiostri e dai luoghi pubblici ${ }^{8}$. Anche quando esse erano più piccole, come è stato già mostrato, si esigeva di tenerle chiuse dentro la casa. Infatti Barberino comparava la ragazza al tesoro perché

\section{e ogni cosa rara}

dice un dicreto ch'è tenuta cara ${ }^{59}$.

La stessa opinione aveva Bernardino quando avvertiva i genitori di allontanare le loro figlie dalle finestre, perché esse contavano di incontrare qualcuno per chiacchierare, sentire i canti oppure per osservare i giovani giocare a palla ${ }^{60}$. Questa ammonizione di non stare presso le finestre e le porte, dimostra che questo era un vizio molto frequente tra le donne di quel tempo. Per tenerle lontane da questi posti esse dovevano essere sempre occupate. Infatti il lavoro delle giovani donne dentro le case era sempre ben visto, ma dipendeva dallo status sociale della famiglia. Invero le ragazze delle case nobili, secondo Barberino, dovessero saper fare qualcosa, come ad esempio cucire e ricamare, perché della fortuna non si può mai essere sicuri ${ }^{61}$.

Quando capitava alle ragazze uscire di casa il poeta le avvertiva di comportarsi in modo corretto, di camminare con piccoli passi davanti alla madre e di non guardare d'intorno ${ }^{62}$. In questa età però secondo Barberino le ragazze dovevano rimanere chiuse per non essere viste dagli uomini giovani e per evitare ogni contatto con loro. Il poeta sottolineava che ella già non puote né si conviene a lei l'andare in chiesa ${ }^{63}$. Infatti quando tutta la famiglia andava per la messa esse rimanevano nelle loro stanze e se leger savesse l'uficio suo, che breve dicesse $e^{64}$. Barberino era però contrario alle lunghe preghiere e meditazioni, infatti aggiunge... ben vo' dir che non mi piace ancora ch'ella troppo stia in orazione, però ch'è meglio assai orar fervente e poco ${ }^{65}$.

Quando la ragazza si trovava a un banchetto o nel luogo dove c'era la gente non poteva, secondo il poeta, accompagnare con il sorriso lo sguardo, né insistere nel guardare ${ }^{66}$. Infatti

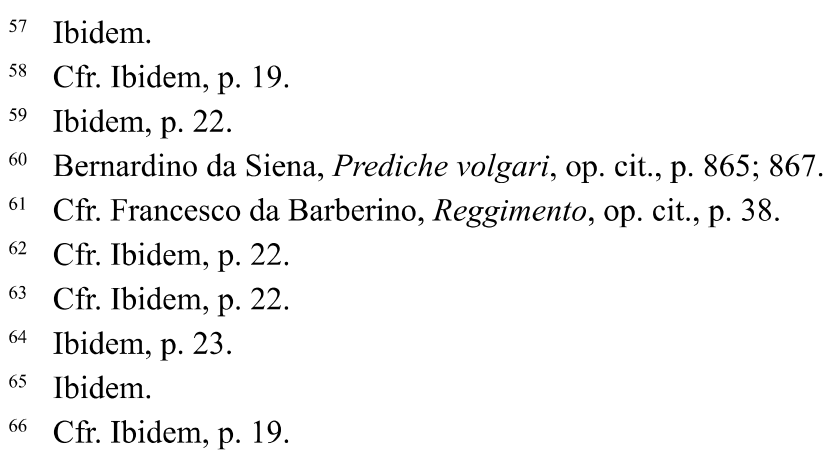


se per volontade di suo padre o di sua madre la convenga venire e fra la gente stare-overo in casa, overo in un giardino, overo ancora forse caminando sovra alcuna cariera, oforse in nave, como tal fiata a queste grandi adviene -, cotanto e non più lievi li occhi suoi ${ }^{67}$. Per loro sicurezza le ragazze dovevano sempre stare in compagnia o della madre o delle maestre $^{68}$ e parlare soltanto quando veniva dato loro il permesso ${ }^{69}$. Invero l'unico posto dove la ragazza poteva parlare tranquillamente era la sua stanza: lì porrà, per suo passare, parole belle e più alquante dire ${ }^{70}$.

Riguardo alla musica Barberino dava loro il permesso di imparare a suonare qualche strumento onesto e bello, però lo dovevano imparare da donna la qual se vien di fuori, tenga con seco allo suo insegnare presente alcuna donna delle sue maestre ${ }^{71}$.

\section{Conclusione}

Il presente studio descrive brevemente l'istruzione delle ragazze vissute nell'epoca medioevale. Sono stati presentati tre modi nei quali le figlie di famiglie nobili potevano ricevere un'educazione adeguata al loro casato. In primo luogo sono state elencate le scuole dei conventi, poi la corte reale o la casa di qualche grande signora o infine la casa propria. Gli scritti pervenuti ai nostri giorni permettono di vedere quale virtù si aspettava la società dalle ragazze nobili per le quali era prevista la vita matrimoniale.

Il Reggimento e costumi di donne scritto da Francesco da Barberino è uno di pochi testi di galateo femminile d'epoca medievale. Il poeta dimostra quali costumi doveva avere la bambina e poi la fanciulla in età di matrimonio che proveniva da famiglia di alto status sociale. Grazie alle sue parole si può vedere quali virtù dovevano avere le ragazze, quali erano i comportamenti ben visti e invece quali non accettabili dalla società del tempo.

\section{Francis from Barberino and the education of girls at the age of marriage Summary}

Francis from Barberino is an author of one of the few female etiquette texts composed in the Middle Ages. In this period, writers wrote some texts about dress etiquette but the most of them was dedicated to the male reader and there are only some text written for women.

In his works, Barberino shows the dress etiquette appropriate for a girl at the age of marriage coming from a family of high social status. Thanks to his work, it is possible to see which virtues was appreciated, what was allowed and which behaviors was not accepted by the society of that time.

Keywords: Francis of Barberino, costumes, regiment, Middle Ages, education

\footnotetext{
Ibidem, p. 20.

68 Cfr. Ibidem, p. 20.

${ }^{69}$ Cfr. Ibidem.

70 Ibidem.

71 Ibidem.
} 


\section{Francesco z Barberino i edukacja dziewcząt $w$ wieku odpowiednim do małżeństwa}

\section{Abstrakt}

Francesco z Barberino jest autorem jednego z niewielu tekstów średniowiecznych skierowanych do kobiet, które dotyczącą zasad zachowania. W tamtym czasie autorzy napisali kilka utworów dotyczących ogólnych zasad zachowania, ale ich odbiorcami byli głównie mężczyźni, natomiast niewiele $\mathrm{z}$ nich było adresowanych do kobiet.

W swoim dziele Barberino przedstawił zasady zachowania, które obowiązywały dziewczęta w wieku stosownym do zamążpójścia, które pochodziły z rodzin znajdujących się na wysokich szczeblach hierarchii społecznej. Dzięki dziełu Francesca z Barberino można dowiedzieć się, jakie cnoty były doceniane, co było dozwolone, a jakie zachowanie nie było akceptowane przez społeczeństwo tamtej epoki.

Nota o Autorze: dr Anna Głusiuk, adiunkt w Katedrze Średniowiecza w Instytucie Nauk Historycznych i Społecznych Uniwersytetu Kardynała Stefana Wyszyńskiego. Zainteresowania naukowe: literatura średniowieczna, sytuacja i rola kobiety w epoce średniowiecza. 